\title{
Transient Coherent Synchrotron Radiation in Magnetic Bending Systems
}

\author{
R. Li, C. L. Bohn, and J. J. Bisognano \\ Thomas Jefferson National Accelerator Facility, Newport News, VA 23606
}

\begin{abstract}
Transient evolution of the power radiated coherently by a charged-particle bunch orbiting between two infinite, parallel conducting plates is calculated. The plates comprise an idealized vacuum pipe in a bending magnet. The bunch moves on a trajectory such that it suddenly diverts from a straightline path to a circular orbit and begins radiating. The influence of the plates on the transients is contrasted to their shielding of the steady-state radiated power. The effect of the radiation field on beam emittance in a magnetic bending system is also quantified.
\end{abstract}

PACS numbers: 29.27.Bd, 41.20.Bt, 41.60.Ap, 41.85.Lc 
Coherent synchrotron radiation (CSR) is a longstanding topic of study [1]. When accelerated, a compact ensemble, or bunch, of relativistic charged particles radiates coherently at wavelengths comparable to or longer than the bunch length. Coherence greatly enhances the radiated power above that of incoherent synchrotron radiation, as observed in laboratory experiments $[2]$ :

Recent developments in electron-gun and injector technologies enable production of short (mm-length), high-charge (nC-regime) bunches [3]. Therefore one can conceive of compact synchrotrons for the production of intense mm-wavelength radiation, and a synchrotron of this type has recently been proposed [4]. Short, high-charge bunches are also of interest in high-power free-electron lasers, damping rings and bunch compressors for linear colliders, and recirculating accelerators of high-brightness electron beams [5]. In these machines, and generally also in storage rings, the beamline will normally incorporate arrays of bending magnets to transport the beam and manipulate the bunch length. Their designs must circumvent the possible deleterious effects of CSR which establishes an electromagnetic wake that interacts back on the bunch and distorts it. The distortion might show up as bunch lengthening which will cut off or reduce CSR [6], or as transverse emittance growth [7]. CSR establishes an electromagnetic-field gradient across the bunch and causes the energies of the individual particles comprising the bunch to change by differing amounts as the bunch traverses the bending system. In turn, even if the bending system is achromatic, the transverse emittance of the bunch grows as it moves downstream.

The canonical treatment of CSR entails calculating the steady-state radiation from a rigid bunch that has been executing a circular orbit over an infinite time. The most comprehensive work of this ilk is that of Warnock and collaborators who solve Maxwell's equations in the frequency domain in terms of Bessel functions subject to the boundary conditions of a smooth torus with rectangular cross section [6,8]. A fundamentally important result is that the torus will suppress steady-state $\operatorname{CSR}$ if $\left(h / \pi \sigma_{s}\right)^{2}(w / R) \ll 1$, where $h$ and $w$ are the chamber's height and width, respectively, $R$ is the orbit radius, and $\sigma_{s}$ is the rootmean-square (rms) bunch length. This condition derives from a calculation of the minimum 
frequency at which there is a chamber mode that is both resonant and synchronous with the orbiting bunch. When it is violated there should be appreciable CSR in the wavelength interval $2 \pi \sigma_{s} \lesssim \lambda \lesssim 2 h(w / R)^{1 / 2}[9]$.

In a bend spanning a finite length, the bunch-excited wakefield and its effect back on the bunch are inherently transient phenomena, and analyses restricted to steady-state CSR do not directly apply. Wingham [10] considers transient radiation in a finite bend using a frequency-domain analysis; however, he restricts his analysis to free space. By contrast, we treat transient CSR from a bunch in a finite bend using a time-domain analysis that combines a generalized scalar potential with an infinite-parallel-plate model of the vacuum pipe [11]. The plates are separated by spacing $h$. The bunch moves along the midplane on a straight path during times $-\infty \leq t<0$ and on a circular orbit of radius $R$ during $t \geq 0$. Transients generated by entry of the bunch into the bend act over very short bend angles $\theta \sim \sigma_{s} / R$ and are ignored.

The generalized potential at point $\mathrm{r}$ and time $t$ accounts for the single-particle interaction with the bunch:

$$
\mathcal{V}(\mathbf{r}, t)=e^{2} \int_{-\infty}^{t} d t^{\prime} \int \frac{d \mathbf{r}^{\prime}}{c \tau}\left(1-\beta \cdot \beta^{\prime}\right) \delta\left(t-t^{\prime}-\tau\right) n\left(\mathbf{r}^{\prime}, t^{\prime}\right)
$$

where $e$ is the electron charge, $\beta$ is the velocity divided by the speed of light $c, \tau=\left|\mathbf{r}-\mathbf{r}^{\prime}\right| / c$, $n(\mathbf{r}, t)$ is the number density in the bunch, and primed quantities correspond to the past. This potential combines the scalar $(\Phi)$ and vector $(\mathbf{A})$ potentials derived from the retarded Green's function as $\mathcal{V}=e(\Phi-\beta \cdot \mathbf{A})$, and it determines the relativistic Lagrangian [12]. The power radiated by a single electron is simply the negative of its rate of energy change, i.e., $-\partial \mathcal{V} / \partial t$. For a charge distribution moving in free space along our model trajectory, it is given by

$$
P(\mathbf{r}, t)=-e^{2} \int_{0 \leq \tau \leq t} \frac{d \mathbf{r}^{\prime}}{c \tau}\left(1-\beta \cdot \beta^{\prime}\right) \frac{\partial}{\partial t} n\left(\mathbf{r}^{\prime}, t-\tau\right) .
$$

The total power radiated by the entire bunch is $P_{\text {tot }}(t)=\int d \mathbf{r} P(\mathbf{r}, t) n(\mathbf{r}, t)$. Taking $n(\mathbf{r}, t)$ to be continuous implicitly phase-averages over the incoherent radiation and thereby retains 
only CSR and the space-charge force. We dropped the contribution from the straight-path motion during $t<0$ because it is coulombic, not radiative, and is important only locally, for. example, at the beginning of the circular trajectory [13]. Our focus is on the effects of the global radiative interaction and we will be discarding coulomb terms everywhere along the trajectory.

Our goal is to quantify the radiated power and the influence of CSR on transverse emittance. We use a rigid-line Gaussian bunch as our model charge distribution. The motion of its center for $t \geq 0$ is given by $\theta=\omega_{0} t$, where $\omega_{0}=\beta c / R$ is its orbital frequency. The line density is $n(s, t)=N \lambda(s)$, in which $N$ is the number of particles in the bunch, $\lambda(s)$ is the longitudinal profile, and $s=R\left(\theta-\omega_{0} t\right)$ is the distance measured from the bunch center. The conducting plates establish an infinite array of image bunches moving in free space simultaneously with the real bunch [14]. Each radiated photon that bounces from plate to plate and eventually interacts back on the bunch can be regarded as originating from one of the image bunches at an earlier time. The photon's path length matches the distance between the point of interaction and the point of emission from the image bunch. By formulating the problem this way, we can synthesize and generalize techniques used in recent investigations of steady-state CSR $[15,16]$.

The travel distance of a photon emitted from the $n$th image charge at the source point $\theta^{\prime}$ to the observation point $\theta$ is denoted $c \tau_{n}$. Since the bunch starts the bend at $t=0$, for an electron at $(\theta, t)$ to interact with the $n$th image charge at $\theta^{\prime}$, causality requires $0 \leq c r_{n}(\xi)=\left[(n h)^{2}+4 R^{2} \sin ^{2}(\xi / 2)\right]^{1 / 2} \leq c t$, where $\xi=\theta-\theta^{\prime}$. The power radiated by a single electron is $P(s, t)=\sum P_{n}(s, t)$, where $P_{n}$ is the contribution from the $n$th image bunch:

$P_{n}(s, t)=N e^{2} \beta(-)^{n+1} \int_{\xi_{n}^{-}(t)}^{\xi_{n}^{+}(t)} \frac{1-\beta^{2} \cos \xi}{\tau_{n}(\xi)} \frac{d \lambda(s-\Delta s)}{d \Delta s}$.

Here, $\Delta s(\xi)=R \phi(\xi)$, with $\phi$ being the intrabunch angular spacing between the observation particle with respect to the source particle: $\phi \equiv\left(\theta-\omega_{0} t\right)-\left(\theta^{\prime}-\omega_{0} t^{\prime}\right)=\xi-\omega_{0} \tau_{n}(\xi)$. Positive values of $\phi$ correspond to interactions of trailing particles on leading particles, and vice versa. The integration limits $\xi_{n}^{ \pm}(t)$ follow from causality. For the $n$th image charge at $t^{\prime}=0$ to influence 
the source charge, $t$ has to satisfy $c t \geq n h$. For $n h \leq c t \leq\left[(n h)^{2}+(2 R)^{2}\right]^{1 / 2}$, the integration limits are found by setting $\tau_{n}(\xi)=t$. Taking $\xi_{n}(t)=2 \sin ^{-1}\left\{\left[(c t)^{2}-(n h)^{2}\right]^{1 / 2} /(2 R)\right\}$ with $0 \leq \xi_{n}(t) \leq \pi$, the integration limits are then $\xi_{n}^{ \pm}(t)=2 k \pi \pm \xi_{n}(t)$ for any integer $k$. For $c t>\left[(n h)^{2}+(2 R)^{2}\right]^{1 / 2}$, the time is long enough for a photon emitted by the $n$th image charge at $t^{\prime}=0$ to reacti any point on the circular orbit of the source charge, and $\xi_{n}^{ \pm}(t)= \pm \infty$.

The power radiated by the entire bunch is $P_{\text {tot }}(t)=\sum P_{n}(t)$, where $P_{n}(t)=$ $\int_{-\infty}^{\infty} d s \lambda(s) P_{n}(s, t)$. For a Gaussian profile, after change of variable from $\xi$ to $\phi$,

$$
\begin{aligned}
P_{n}(t) & =\frac{N^{2} e^{2} \beta^{2} c R}{4 \sqrt{\pi} \sigma_{s}^{3}}(-)^{n+1} \int_{\phi_{n}^{-}(t)}^{\phi_{n}^{+}(t)} d \phi \phi f_{n}(\phi) \exp \left(-\frac{\phi^{2}}{4 \sigma_{\phi}^{2}}\right) \\
f_{n}(\phi) & =\frac{1-\beta^{2} \cos \xi_{n}(\phi)}{\xi_{n}(\phi)-\phi-\beta^{2} \sin \xi_{n}(\phi)}
\end{aligned}
$$

in which $\sigma_{\phi}=\sigma_{s} / R$, and $\phi_{n}^{ \pm}(t)=\xi_{n}^{ \pm}(t)-\omega_{0} t$.

The free-space radiated power corresponds to $n=0$, in which case $\phi=\xi-2 \beta|\sin (\xi / 2)|$. Iogansen and Rabinovich [1] show that the radiative interaction force for $\xi \geq 0$ is $\sigma_{\phi}^{-4}$ greater than for $\xi<0$. Hence we consider only $\xi \geq 0$ and consequently, for $\beta \approx 1$, have $\phi \simeq \xi / 2 \gamma^{2}+\xi^{3} / 24 \geq 0$, with $\gamma \equiv\left(1-\beta^{2}\right)^{-1 / 2}$. We neglect the local space-charge interaction in favor of the global radiative interaction and take $\phi \simeq \xi^{3} / 24$. This approximation holds when $\xi \gg 2 \sqrt{3} / \gamma$, or $\phi \gg 2 \sqrt{3} / \gamma^{3}$. In turn, we have $f_{0} \simeq 2 /(3 \phi)^{1 / 3}$, and from Eq. (4) with $\phi_{0}^{-}(t)=0$

$$
P_{0}(t) \simeq \frac{c N^{2} e^{2}}{\left(6 R^{2} \sigma_{s}^{4}\right)^{1 / 3} \sqrt{\pi}} \gamma\left\{5 / 6,\left[\phi_{0}^{+}(t)\right]^{2} / 4 \sigma_{\phi}^{2}\right\}
$$

where $\gamma(\mu, x)$ is an incomplete Gamma function, and $\phi_{0}^{+}(t)=\left[\xi_{0}^{+}(t)\right]^{3} / 24$, in which $\xi_{0}^{+}(t)=$ $2 \sin ^{-1}\left(\omega_{0} t / 2 \beta\right)$. The formation length $L$ for the radiated power to saturate to steady state corresponds to the time for a photon to travel from the bunch's tail to its head, for which $\phi_{0}^{+} \sim 4 \sigma_{\phi}$, yielding $L \sim 2 \beta\left(12 R^{2} \sigma_{s}\right)^{1 / 3}$. The steady-state power loss in free space is

$$
P_{0}(\infty)=\frac{c N^{2} e^{2}}{\left(R^{2} \sigma_{s}^{4}\right)^{1 / 3}} \frac{3^{1 / 6}}{2 \pi}[\Gamma(2 / 3)]^{2}
$$

in agreement with Schiff [1]. 
To find the power radiated in the presence of the parallel plates, we use $P_{0}(t)$ from Eq. (5), numerically integrate Eq. (4) to get $P_{n \neq 0}(t)$, and sum over $n$. Representative results appear in Fig. 1 in which the instantaneous radiated power, normalized by the free-space steady-state radiated power, is plotted as a function of bend angle. Parameters in Fig. 1(a) correspond to large vacuum chamber and short bunch, i.e., little shielding of the steadystate radiation. The localized transient peaks are due to the interaction of the bunch with the image charges, and the steady-state power loss is nearly identical with or without the plates. By contrast, parameters in Fig. 1(b) correspond to smaller vacuum chamber and longer bunch, i.e., considerable shielding of the steady-state radiation. Again, there are localized transient peaks. The shielded steady-state power loss is $25 \%$ of that of free-space, a result that agrees with the frequency-domain analysis of Nodvick and Saxon [1]. With lossy plates the transient peaks in Fig. 1 would damp faster to the steady state, and the effect of the walls on steady-state shielding would be reduced. With "black" plates the CSR effect would be the same as in free space.

One can also expand $f_{n \neq 0}(\phi)$ in a Taylor series. If $(n h / R)^{3 / 2} \gg \sigma_{\phi}$, the leading term dominates, and thus $P_{n \neq 0}(t)$ can be obtained explicitly and used to estimate all the features of the transient peaks appearing in Fig. 1: for $\theta_{c} \equiv 12^{1 / 4}(n h / R)^{1 / 2}<1$, the center angle is $\simeq \theta_{c}$, the width is $\simeq 4 \sqrt{3}\left(\sigma_{s} / n h\right)$, and the amplitude (ratioed to free space) is $\simeq 3\left[\sigma_{\phi} /(n h / R)^{3 / 2}\right]^{1 / 3}$. However, if the inequality is not satisfied, $f_{n \not p 0}(\phi)$ is very nonlinear, and one has to retain many terms in the Taylor expansion. Terms with odd powers of $\phi$ contribute to steady-state shielding.

We now consider effects of CSR on the normalized transverse rms emittance $\epsilon$ of the beam in a bending system. By definition, $\epsilon \equiv \gamma_{0}\left[\left((x-\langle x\rangle)^{2}\right\rangle\left\langle\left(x^{\prime}-\left\langle x^{\prime}\right\rangle\right)^{2}\right\rangle-\left\langle(x-\langle x\rangle)\left(x^{\prime}-\left\langle x^{\prime}\right\rangle\right)\right\rangle^{2}\right]^{1 / 2}$, in which $\gamma_{0}$ is the design energy, $x$ is the offset from the design orbit, the prime denotes differentiation with respect to the bunch's trajectory coordinate, and the averages are taken over the whole bunch.

Recent investigations have generated a scaling relation which, taken at face value, predicts potentially disastrous emittance growth in short, high-charge bunches [7]: 


$$
\Delta \epsilon \simeq 0.5 \theta^{2} \sigma_{x} \frac{I_{p}}{I_{A}}\left(\frac{R}{\sigma_{s}}\right)^{1 / 3}
$$

in which $\theta$ is the total bend angle, $\sigma_{x}$ is the rms beam size in the bend plane, $I_{p}$ is the peak current, and $I_{A}=17 \mathrm{kA}$ is the Alfven current. This is derived for free-space steady-state conditions and has been applied directly to bends of arbitrary length. A second emittancegrowth mechanism, due to "centrifugal space-charge force" acting locally within the bunch, has been predicted by Carlsten and Raubenheimer [17]. According to their estimate, apart from a logarithmic factor, the ratio of the emittance growth from this effect to that from CSR is about $\left(\sigma_{x} / \sigma_{s}\right)\left(\sigma_{s} / R\right)^{1 / 3}$. When this ratio is small compared to unity, CSR is of principal concern. In fact, our line-charge model is valid provided $\sigma_{r} / \sigma_{s} \ll\left(R / \sigma_{s}\right)^{1 / 3}$, with $\sigma_{\tau}$ denoting the beam radius [15], which means emittance growth from CSR dominates that from the centrifugal space-charge force whenever the line-charge model applies. Because the scaling relation (7) predicts severe degradation for short bunches $\left(\propto \sigma_{s}^{-4 / 3}\right)$ and long bends $\left(\propto \theta^{2}\right)$, revising it to include transient CSR and beam transport in the bending-system lattice is important.

The calculation of $\epsilon$ proceeds directly from the equation of motion. We assume the rigid-line-charge model is valid and the longitudinal CSR force is the main cause of emittance growth. To first order in deviations from the design orbit, the equation of motion for a particle in the relativistic bunch is $d^{2} x / d t^{2}+\omega_{0}^{2} x=\left(R \omega_{0}^{2} / \gamma_{0}\right)\left[\gamma_{1}(s, 0)+\Delta \gamma(s, t)\right]$, in which $x=r-R$ is the offset from the design orbit in the bend plane, $\gamma_{1}(s, 0)$ is the initial energy offset from the design energy $\gamma_{0}$, and $\Delta \gamma(s, t)$ is the CSR-induced energy change: $\Delta \gamma(s, t) m c^{2}=-\int_{0}^{t} d t^{\prime} P\left(s, t^{\prime}\right)$. For example, in free space, $\Delta \gamma(s, t)=\kappa \int_{0}^{t} \omega_{0} d t^{\prime} g\left(s, t^{\prime}\right)$ with $\kappa \equiv\left[2 /\left(3^{1 / 3} \sqrt{2 \pi}\right)\right]\left(I_{p} / I_{A}\right)\left(R / \sigma_{s}\right)^{1 / 3}$, and

$$
g(s, t)=\int_{0}^{\phi_{0}^{+}(t)} \frac{d \phi}{\phi^{1 / 3}}\left(\phi_{s}-\phi\right) e^{-\frac{1}{2}\left(\phi_{s}-\phi\right)^{2}}
$$

with $\phi_{s}=s / \sigma_{s}$ is the normalized force. Solving the equation of motion with Green's theorem yields 


$$
\left(\begin{array}{c}
x(s, t) \\
x^{\prime}(s, t)
\end{array}\right)=M\left[\left(\begin{array}{c}
x(s, 0) \\
x^{\prime}(s, 0)
\end{array}\right)+\left(\begin{array}{c}
\Delta x(s, t) \\
\Delta x^{\prime}(s, t)
\end{array}\right)\right]+D \frac{\gamma_{1}(s, 0)}{\gamma_{0}},
$$

where the prime denotes differentiation with respect to $R \omega_{0} t, M$ and $D$ are the transport matrix and dispersion vector for the bend, which are, respectively,

$M=\left(\begin{array}{cc}\cos \omega_{0} t & R \sin \omega_{0} t \\ -\sin \omega_{0} t / R & \cos \omega_{0} t\end{array}\right), D=\left(\begin{array}{c}R\left(1-\cos \omega_{0} t\right) \\ \sin \omega_{0} t\end{array}\right)$,

and $\left[\Delta x(s, t), \Delta x^{\prime}(s, t)\right]$ denote the CSR-induced dispersive offset and angle, respectively,

$$
\left(\begin{array}{c}
\Delta x(s, t) \\
\Delta x^{\prime}(s, t)
\end{array}\right)=\int_{0}^{t} \omega_{0} d t^{\prime}\left(\begin{array}{c}
-R \sin \omega_{0} t^{\prime} \\
\cos \omega_{0} t^{\prime}
\end{array}\right) \frac{\Delta \gamma\left(s, t^{\prime}\right)}{\gamma_{0}} .
$$

The emittance satisfies

$$
\epsilon^{2}=\gamma_{0}^{2}\left|\begin{array}{cc}
\left\langle\left(x_{0}+\delta x\right)^{2}\right\rangle & \left\langle\left(x_{0}+\delta x\right)\left(x_{0}^{\prime}+\delta x^{\prime}\right)\right\rangle \\
\left\langle\left(x_{0}+\delta x\right)\left(x_{0}^{\prime}+\delta x^{\prime}\right)\right\rangle & \left\langle\left(x_{0}^{\prime}+\delta x^{\prime}\right)^{2}\right\rangle
\end{array}\right|+[\cdots]\left\langle\gamma_{1}^{2}\right\rangle,
$$

where $x_{0}, x_{0}^{\prime}$ are initial conditions, $\delta x=\Delta x-\langle\Delta x\rangle$ and $\delta x^{\prime}=\Delta x^{\prime}-\left\langle\Delta x^{\prime}\right\rangle$. The last term, related to the initial energy spread, is zero in an achromatic bending system.

The general solution (11) is the key for revising the scaling relation (7). It can be written as $\epsilon^{2}=\epsilon_{0}^{2}+(\Delta \epsilon)^{2}$, with $\epsilon_{0}$ the initial emittance defined in terms of $x_{0}, x_{0}^{\prime}$ and $\Delta \epsilon$ the CSR-induced emittance growth. In the special case that $\left\langle\delta x^{2}\right\rangle /\left\langle x_{0}^{2}\right\rangle, R\left\langle x_{0} x_{0}^{\prime}\right\rangle /\left\langle x_{0}^{2}\right\rangle$, and $R^{2}\left\langle x_{0}^{\prime 2}\right\rangle /\left\langle x_{0}^{2}\right\rangle$ are all much smaller than unity, then $\Delta \epsilon \simeq \gamma_{0}\left[\left\langle x_{0}^{2}\right\rangle\left\langle\delta x^{\prime 2}\right\rangle\right]^{1 / 2}$. Eq. (7) is predicated on this special case with $\left[\left\langle\delta x^{2}\right\rangle\right]^{1 / 2} \simeq 0.5 \kappa\left(\omega_{0} t\right)^{2}$. However, using Eqs. (10) and (8) with the free-space steady-state condition $g(s, t)=g(s ; \infty)$ yields $\left[\left\langle\delta x^{\prime 2}\right\rangle\right]^{1 / 2}=0.4\left(\omega_{0} t \sin \omega_{0} t+\right.$ $\left.\cos \omega_{0} t-1\right)$, indicating a $\theta \sin \theta$-dependence instead of $\theta^{2}$ for large bend angle $\theta=\omega_{0} t \gg 1$.

General aspects of the emittance calculation with conducting walls can be gleaned from Fig. 1. First, in short bends, the existence of the formation length to reach steady state will cause the emittance growth to be less then that predicted for steady-state radiation. Second, in long bends, the transients will tend to average out and the steady-state radiation field will predominate. However, for long bending systems, the scaling with bend angle will be weaker than quadratic, as indicated above. 
In summary, we have calculated analytically the transient radiated power due to coherent synchrotron radiation from a charged-particle bunch in a bending magnet. We have also calculated analytically how the electromagnetic force on the particles in the bunch due to CSR degrades its transverse emittance. Transient effects and proper treatment of the transport lattice appear to reduce significantly the emittance growth compared to that calculated by overlooking these details.

The theory bears on an important question regarding high-brightness accelerators: How should bending systems be designed to preserve beam quality? The present treatment points to a key element of the strategy, namely, keeping the bunch length long in the bending magnets. When one must manipulate short bunches through a bending system, such as in a magnetic bunch compressor, bending magnets that are short compared to the CSR formation length are preferred. However, this criterion may not be sufficient. The potential importance of intermagnet coupling has yet to be established quantitatively. The methods of this paper indicate that the radiation field will almost comove with a bunch through straight connecting sections, thereby continually increasing the energy spread in the bunch, but it also will decrease in amplitude as the distance from the source point increases.

Though we ignored finite transverse beam size, space charge, and dynamical changes in bunch length, the Lagrangian with the generalized potential given in Eq. (1) includes all three. It comprises the recipe for calculating interparticle forces in numerical simulations of beam dynamics in bends. A second application of our approach is the deciphering of spectral information from CSR-based diagnostics involving a magnetic bend [18]. Extending the transient formalism to the frequency domain may enable use of these diagnostics for accurate absolute measurements of very short bunch lengths.

We thank J. Delayen and H. Liu for stimulating discussions that scoped this work in its early stages. Delayen asked probing questions about transients, and Liu pointed out that quadratic dependence of emittance on bend angle is only valid for short bends. We also thank B. Yunn for carefully reviewing the manuscript. This work was supported by the U. S. Department of Energy under contract DE-AC05-84ER40150. 


\section{REFERENCES}

[1] Early papers include L. I. Schiff, Rev. Sci. Instr. 17, 6 (1946); J. S. Nodvick and D. S. Saxon, Phys. Rev. 96, 180 (1954); L. V. Iogansen and M. S. Rabinovich, Sov. Phys. JETP 37, 83 (1960); P. Goldreich and D. A. Keeley, Ap. J. 170, 463 (1971).

[2] T. Nakazato et al., Phys. Rev. Lett. 63, 1245 (1989).

[3] The state of electron-gun and injector technologies is summarized by related papers in Microbunches Workshop, edited by E. B. Blum, M. Deines, and J. B. Murphy, AIP Conf. Proc. No. 367, (AIP, Woodbury, NY, 1996).

[4] J. B. Murphy and S. Krinsky, Nucl. Instrum. and Methods of Phys. Res. A346, 571 (1994).

[5] C. L. Bohn, in Microbunches Workshop (Ref. [3]), pp. 391-396.

[6] R. L. Warnock and K. Bane, Stanford Linear Accelerator Center Report No. SLACPUB-95-6837, 1995 (unpublished).

[7] T. O. Raubenheimer, in Microbunches Workshop (Ref. [3]), pp. 94-106.

[8] R. L. Warnock and P. Morton, Part. Accel. 25, 113 (1990); K.-Y. Ng and R. L. Warnock, Phys. Rev. D 40, 231 (1989).

[9] R. L. Warnock, in Proceedings of the 1991 Particle Accelerator Conference, edited by L. Lazama and J. Chew, (IEEE, Piscataway, NJ, 1991), pp. 1824-1826.

[10] D. J. Wingham, Phys. Rev. D 35, 2584 (1987).

[11] Warnock and Bane, (Ref. [6]), find that the steady-state wake voltage for a beam circulating in the midplane between two infinite parallel plates is nearly the same as that in a torus, especially near the beam centroid.

[12] J. D Jackson, Classical Electrodynamics, (John Wiley, New York, NY, 1962), pp. 404409; R. Becker, Electromagnetic Fields and Interactions, (Blaisdell, New York, NY, 
1964), pp. 325-327.

[13] The straight-path electromagnetic field is given in J. D. Jackson, (Ref. [12]), p. 381, and is appreciable only for $\omega_{0} t \lesssim 1 / \gamma^{2}$, i.e., early in the bend.

[14] It is straightforward to show that superposing the free-space Green's function of a source charge and its associated array of image charges is equivalent to using the Green's function of the source charge with the parallel-plate boundary condition.

[15] Ya. S. Derbenev et al., DESY Report No. TESLA-FEL-95-05, 1995 (unpublished).

[16] J. B. Murphy, S. Krinsky, and R. L. Gluckstern, in Proceedings of the 1995 Particle Accelerator Conference, (IEEE, Piscataway, NJ, 1991), pp. 2980-2982.

[17] B. E. Carlsten and T. O. Raubenheimer, Phys. Rev. E 51, 1453 (1995).

[18] D. X. Wang et al., in Microbunches Workshop (Ref. [3]), pp. 502-511. 


\section{FIGURES}

FIG 1. The dotted curves denote the transient free-space power $P_{0}(t) / P_{0}(\infty)$ radiated by a bunch in a bend versus bend angle $\theta=\omega_{0} t$. The solid curves denote the transient radiated power $P_{\text {tot }}(t) / P_{0}(\infty)$ in parallel conducting plates. Parameters are: $R=1 \mathrm{~m}, \gamma=80$, and (a) $h=0.05 \mathrm{~m}, \sigma_{s}=0.5 \mathrm{~mm}$ (formation angle $=21^{\circ}$ ); (b) $h=0.02 \mathrm{~m}, \sigma_{s}=1.0 \mathrm{~mm}$ (formation angle $=26^{\circ}$ ). 


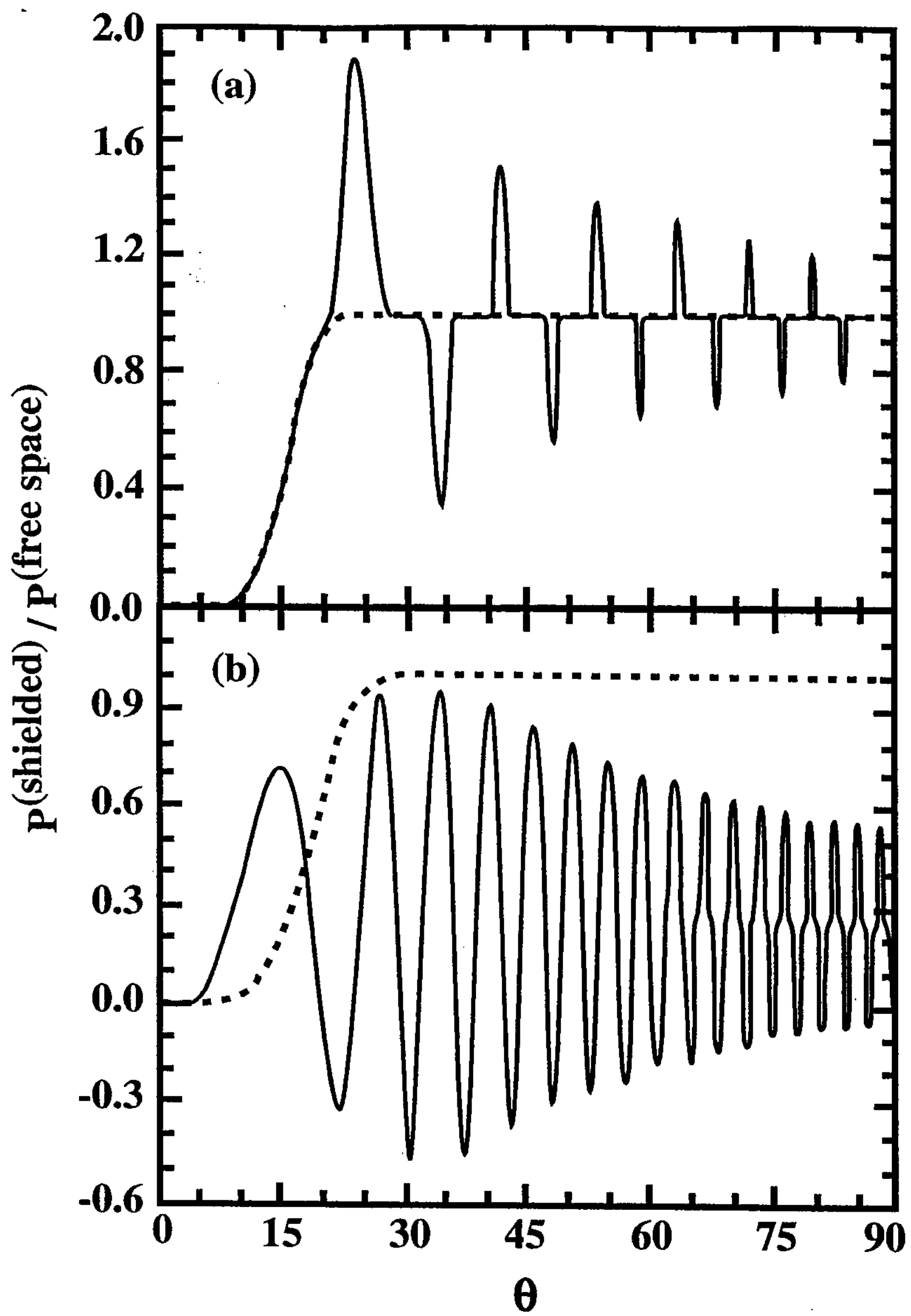

